\title{
Collaborative learning for classification and prediction of building energy flexibility
}

\author{
Anil Kumar*, Elena Mocanu ${ }^{\dagger *}$, Muhammad Babar ${ }^{\ddagger}$, Phuong H. Nguyen ${ }^{\S *}$ \\ *Department of Electrical Engineering, Eindhoven University of Technology, 5600MB Eindhoven, The Netherlands \\ ${ }^{\dagger}$ Department of Computer Science, University of Twente, 7522NH Enschede, The Netherlands \\ ${ }^{\ddagger}$ Digital Grid Operations, DNV-GL Energy, Arnhem, 6812 AR, The Netherlands \\ $\S$ Luxembourg Institute of Science and Technology (LIST), 4422 Belvaux, Luxembourg \\ a.anil.kumar@student.tue.nl, e.mocanu@utwente.nl, m.babar@dnvgl.com, phuong.nguyen@list.lu
}

\begin{abstract}
In this paper we propose an simple digital learning platform for flexible energy detection using data with fine granularity. The platform is empowered with artificially intelligent methods aiming to quantify the uncertainty of building energy consumption at building level, as well as at the aggregated level. Two major learning tasks are perform in this context: prediction and classification. Firstly, the building energy prediction with various time steps resolution are perform using methods such as Fully Connected Neural Networks (FCNN), Long short-term memory (LSTM), and Decision Trees (DT). Secondly, a Support Vector Machine (SVM) method is used to unlock the building energy flexibility by performing classification assuming three different levels of flexibility. Further on, a collaborative task is integrate within the platform to improve the multi-class classification accuracy. Through the end, we argue that this approach can be considered a solid integrated and automated basic block able to incorporate future AI models in (near) real-time to explore the benefits at the synergy between built environment and emerging smart grid technologies and applications.
\end{abstract}

Index Terms-Artificial Intelligence, Classification, Data analytics, Deep learning, Energy Prediction

\section{INTRODUCTION}

Electrical patterns at the building level are recognized to have a highly non-linear and non-stationary character. In the one hand, the increase in variability is given by the new generation capabilities of the buildings, as well as the most traditional recognized factors to influencing it such as price, meteorological conditions, and so on. On the other hand, a more active engagement of end-user participation, together with emerging smart grid technologies and applications are expected to appear in near future and to add even more variability in the electrical patterns [1] [2]. Aiming to provide flexibility and efficiency in the whole energy system, in this paper, we investigate how the latest artificial intelligence methods can be used to analyze and extract the useful knowledge from highly variable electrical patters. Particularly, using a monitoring platform based on end-to-end modular sensor solution with high sampling rate we proposed a integrated data-driven model for building energy prediction and flexibility detection, coupled with a cooperative learning behavior to increase their accuracy.

Recently, complementary with the most traditional statistical learning approaches, deep learning techniques became popular for energy prediction given their abilities to cope with high non-linear time series. In 2014, the Conditional Restricted Boltzmann Machine (CRBM) [3], and Factored Conditional Restricted Boltzmann Machine machine (FCRBM) [4] [5] were introduced. Later on, deep learning methods such as Long Short-Term Memory (LSTM) [6]-[8], Deep Recurrent Neural Networks (DRNN) [9] and Multi Layer Perceptron (MLP) [10] are introduced and compared with Support Vector Machine [4], Random Forest [11]) and so on. Moreover, their success is acknowledged for different types of important signals in power system [12] (e.g. short-term wind power forecast), while in recent studies the LSTM was used for prediction as an integrated part for a solution to optimize a decentralized renewable energy system.

For the classification problem, plenty of deterministic or probabilistic algorithms are known, where every observation is analyzed into a set of quantifiable properties. Intuitively, a learned classifier should be: based on enough training examples, fit the training example, and simple. One of these algorithms is Support Vector Machine (SVM) [13] which usually reaches a very good accuracy and has a good theoretical framework in comparison with others (e.g. , Gaussian Mixture Model [14], AdaBoost [15], or Random Forest [11]). Thus, we have chosen to use SVM in this work to do the classification. Moreover, the classification can be thought of as two separate problems: binary classification and multiclass classification. As the building energy patterns may consists of various levels of flexibility, in the remaining of this paper we will address the multiclass classification problem.

Our contribution can be summarized as follows:

- we proposed a simple artificially intelligent digital learning platform, called AIDigi platform.

- The AIDigi platform is able to perform two tasks: prediction and multi-class classification. Based on this a collaborative learning task was included to increase their accuracy.

- we showed that in such embodied platform for building energy flexibility provable advances of a broad class of AI methods can be achieved when we took the full advantage of an integrated and automated solution.

The remaining of this paper is organized as follows. In Section II, the problems are formalized and the learning 


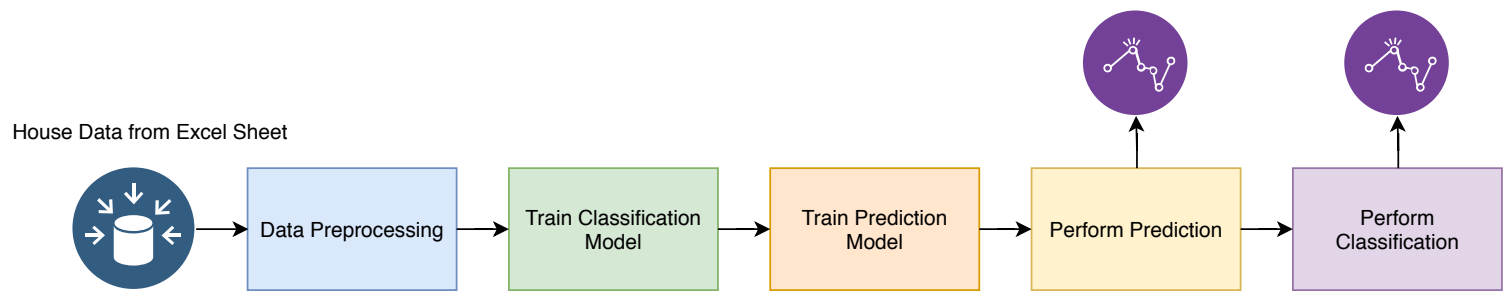

Fig. 1. The general flow diagram of the proposed artificially intelligent digital learning platform

tasks are described. Then, the methods used are presented in Section III, wile the data characteristics are detailed in Section IV. The results are discussed in Section V. Finally, in Section VI the conclusion and further research direction are discussed.

\section{PROBLEM FORMULATION}

Here we define the problems addressed within the AIDigi platform as employed in this work. More formally, let define an input space $\mathcal{X}$ and an output space (label space) $\mathcal{Y}$. Thus, the AIDigi platform is aiming to provide a multi-task solution for two learning problems, as described next.

\section{A. Learning tasks}

Two specifics tasks integrated within the AIDigi platform are Prediction Problem and Classification Problem. Within the prediction task we aim to minimize the expected loss function, defined as the distance $(D)$ between the predict value $\left(\tilde{y}_{t+1}\right)$ and the real value $\left(y_{t+1}\right)$. For this, various distance functions may be used (e.g. $l_{1}$ norm, $l_{2}$ norm, and so on). Thus, we formalize the prediction task as follows

$$
\min \mathrm{D}\left(\tilde{y}_{t+1}, y_{t+1}\right), \quad \forall y \in \mathcal{Y}, \forall t
$$

For a classification problem the question of learning is reduced to the question of estimating a functional relationship of the form $\mathcal{C}: X \rightarrow Y$. Usually, the mapping $\mathcal{C}$ is called a classifier. In order to do this, we get access to some training points $\left(X_{1}, Y_{1}\right), \ldots,\left(X_{n}, Y_{n}\right) \in \mathcal{X} \times \mathcal{Y}$. A classification algorithm is a procedure that takes the training data as input and outputs a classifier $\mathcal{C}$. The learning goal is then to find a $\mathcal{C}$ which makes "as few errors as possible". In our specific case, the $X$ space is given by the building energy consumption and the $Y$ space is given by the level of building flexibility. We assume three classes of load, $l_{(\cdot)}$, based on the peak value at every time step as follow

- Base load, $l_{1} \in[0,40] \%$ of peak value

- Partially flexible load, $l_{2} \in[40,70] \%$ of peak value

- Flexible load, $l_{3}>70 \%$ of peak value

These hard constrains are required as we don't have access to the inside building appliances data. However, the electricity demand profiles were analyzed to gain insight into the potential value of peak-shifting mechanisms and balancing electricity demand with local generation. Without being the scope of this paper, we acknowledge the need of a more sophisticated energy dissagregation module as information from flexible building sub-systems (e.g. heating system, air conditioned system, and so on) will become available.

\section{B. Collaborative AI task}

Considering the joint output of prediction and classification we designed a collaborative learning task in order to improve the overall performance of the platform. This consists in a simple rule which aims to an agreement between the predicted value $(\tilde{y})$ and the specific estimated class $(\tilde{L})$ at moment $t+1$.

$$
\left\{\begin{array}{lll}
\text { if } & \tilde{y}_{t+1} \in \tilde{L}_{t+1}, & \text { keep }\left\{\tilde{y}_{t+1}, \tilde{L}_{t+1}\right\} \\
\text { if } \tilde{y}_{t+1} \notin \tilde{L}_{t+1}, & \text { find a new } \tilde{L}_{t+1}
\end{array}\right.
$$

For example, if the predicted building energy consumption is very small $\left(\tilde{y}_{t+1} \approx 0\right)$ we do not expect to have flexibility. Furthermore, in all tasks we do not make specific assumptions on the spaces $\mathcal{X}$ or $\mathcal{Y}$, but we do make an assumption on the mechanism which generates those training points. Namely, we assume that there exists a joint probability distribution $P$ on $\mathcal{X} \times \mathcal{Y}$ and the training examples $\left(X_{i}, Y_{i}\right)$ are sampled independently from this distribution $P$.

\section{METHODS}

\section{A. The artificially intelligent digital learning platform}

The detailed flow diagram of the proposed AIdigi (artificially intelligent digital learning) platform is presented in Figure 2, where $H_{1}$ and $H_{2}$ represent data of house 1 and 2; $M_{p}$ represents the original matrix (data of all houses) and $M_{c}$ represents the classification data generated in three classes (i.e. $L_{1}, L_{2}$, and $L_{3}$ ). Beside this, the training and testing subsets of data used for prediction $\left(P_{t r}\right.$ and $\left.P_{t e}\right)$ and classification $\left(C_{t r}\right.$ and $C_{t e}$ ) are represented as input to the prediction model, $P M$, and classification model, $C M$, respectively. The link between prediction results and the classification procedure will be further on detailed in the Section III-A0b.

a) Prediction Methods: A comparison between Long short-term memory (LSTM), Fully Connected Neural Networks (FCNN) and Decision Trees (DT) was performed.

b) Classification Methods: Introduced by Cortes and Vapnik [13], Support Vector Machine (SVM) is perhaps the most popular classification method. Independently of the method selection, the output of the multi-class classification method for $N$ classes is a confusion matrix ${ }^{1}, \mathcal{C}^{N \times N}$ for all $N \in \mathbb{N}$, with the left axis showing the true class and the top axis showing the class assigned to an item with that true class. Each element $i, j$ of the matrix would be the number of items with true class $i$ that were classified as being in class $j$. Further on, the instances are typical interpret as the true positive $(t p)$,

\footnotetext{
${ }^{1}$ also known as a contingency table or an error matrix
} 


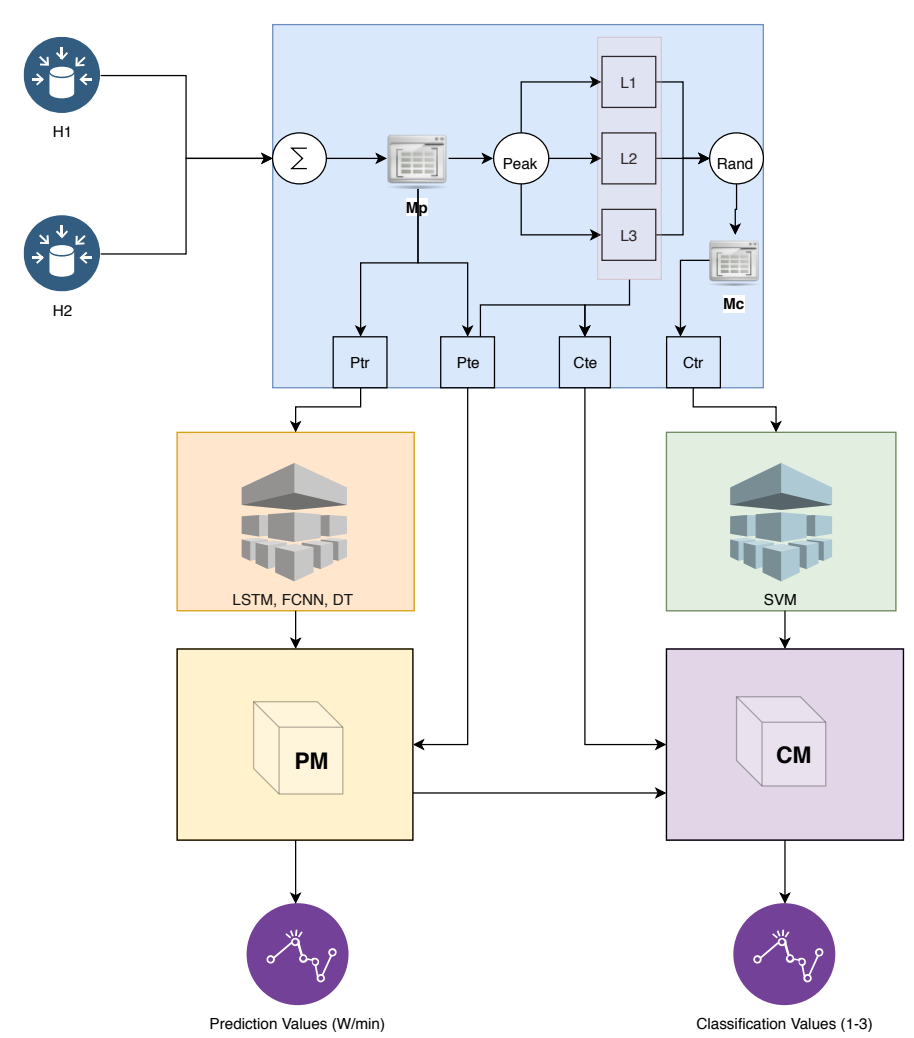

Fig. 2. AIdigi: The detailed flow diagram of the proposed artificially intelligent digital learning platform.

false positive $(f p)$, false negative $(f n)$, and true negative $(t n)$. Thus, inside the $\mathcal{C}^{N \times N}$ matrix these are define by:

$$
\left\{\begin{aligned}
t p_{i} & =\mathcal{C}_{i i}, \quad \forall i \in[1, N] \\
f p_{i} & =\sum_{l=1}^{N} \mathcal{C}_{l i}-t p_{i} \\
f n_{i} & =\sum_{l=1}^{N} \mathcal{C}_{i l}-t p_{i} \\
t n_{i} & =\sum_{l=1}^{N} \sum_{k=1}^{N} \mathcal{C}_{l k}-t p_{i}-f p_{i}-f n_{i}
\end{aligned}\right.
$$

\section{DATA CHARACTERISTICS}

In this paper we have used data from Dutch residential buildings with fine granularity provided by digiEMS, a Dutch start-up company, as part of the UNICORN project. More specifically, we have used 19 full days recorded in the time interval which range from $8^{\text {th }}$ October 2018 to $15^{\text {th }}$ November 2018. From this, we have used the first 18 days for training and one day for testing (the last day).

In order to understand the effects of renewable energy sources on load duration we select and analyze different electrical patterns (i.e. building energy consumption, building energy consumption with PV generation capabilities, and aggregated buildings energy). We have first access the digiEMS company Data acquisition and analysis module and extract the data with less then one minute resolution. To have the exact control of timing, because of the multi-tasking nature of the AIdigi platform, we perform the following data preprocessing steps: i) averaging the data per minute, ii) data normalization, and iii) data interpolation. Further on, in Table I a short summary of the basic data statistics used in this paper are detailed.

TABLE I

BASIC DATA CHARACTERISTICS

\begin{tabular}{lccc} 
& $\begin{array}{c}\text { Peak value } \\
{[\mathrm{W}]}\end{array}$ & $\begin{array}{c}\text { Mean } \\
(\mu)\end{array}$ & $\begin{array}{c}\text { Std. dev. } \\
(\Sigma)\end{array}$ \\
\hline $\begin{array}{l}\text { Building energy consumption } \\
\text { without PV generation }\end{array}$ & 4216.37 & 345.03 & 428.27 \\
\hline $\begin{array}{l}\text { Building energy consumption } \\
\text { with PV generation }\end{array}$ & 4898.00 & 496.80 & 711.84 \\
\hline
\end{tabular}

Overall, we have analyzed in the off-line settings the prediction and classification capabilities of the proposed AIdigi platform using data with 1 minute, 5 minutes, and 15 minutes resolution. The proposed cloud-based solution is aiming for a fast adaptation to the online settings, and thus the prediction and classification procedure are chosen to be performed for each time step ahead individually. The day-ahead analysis includes for example, using data with 1 minute resolution to predict 1440 time steps in the future, using data with 5 minutes resolution to predict 288 time steps in the future and using data with 15 minutes resolution to predict 96 time steps in the future.

\section{NuMERicAl RESUlts}

\section{A. Evaluation metrics to assess the AIdigi platform accuracy}

In general, a large rage of metrics can be used to evaluate the error between input and output values as a generic part of an artificial intelligence environment. The AIdigi platform evaluation uses two metrics for the energy prediction problem and four metrics for the classification problem.

a) Metrics used for prediction assessment: The evaluation of the prediction model is performed using the mean square error (MSE) and mean percentage error (MAE). MSE between input and output values is given by $M S E=$ $\frac{1}{n} \sum_{i=1}^{n}\left(y_{i}-\hat{y}_{i}\right)^{2}$, where $n$ is the amount of data samples, $y_{i}$ is the input data, and $\hat{y}_{i}$ is the predicted output data. Complementary, the mean percentage error (MAE) is the calculated average of percentage errors by which predicted values of a model differ from actual values of the quantity being estimated. Thus we have computed $M A E=\frac{100 \%}{n} \sum_{i=1}^{n} \frac{y_{i}-\hat{y}_{i}}{y_{t}}$, as a slight different evaluation metrics to asses the accuracy of the models which consider the weighted error.

b) Metrics used for classification assessment: In order to characterize as fairly as possible the accuracy of the models proposed to classify the flexible load we have calculated the classifier accuracy as follows:

$$
\text { Accuracy }=\frac{\sum_{i=1}^{N} C_{i i}}{\sum_{i=1}^{N} \sum_{j=1}^{N} C_{i j}}
$$

where $C$ is the confusion matrix (also known as a contingency table or an error matrix), $C_{i i}$ represents the positive true value and the denominator represents the total number of data used in the classification procedure. This quantifies the proportion of the total number of instances that were correctly classified. 
Further on, given our specific building energy patterns and the assumption based on peak value considered for class labeling, we calculate the recall define by Recall $=t p /(t p+f n)$, as well as the precision metrics define by Precision = $t p /(t p+f p)$. Finally, a $F 1$ Score is reported as a weighted average of the true positive rate (recall) and precision.

The need to add more metrics to asses the quality of the classification is given by our special case with imbalanced data sets. In data mining community is it well acknowledge that the confusion-matrix accuracy does not reflect properly the case of imbalanced data sets, and thus is recommended to expand the results analyzes using multiple metrics. In addition to the metrics used in this work, the Balanced Accuracy (bACC) metrics can be used to overcome this problem.

\section{B. Implementation details}

The AIdigi platform was implement in Python. Prerequisite for program execution: Anaconda environment with the following packages: Numpy, Pandas, Keras [16], and sklearn.

Based on a small trial and error test we chose to use the FCNN model with three hidden layers (i.e. $h_{1}=1024$, $h_{2}=2048$ and $\left.h_{3}=4096\right)$ in the case of data with one minute resolution, and (i.e. $h_{1}=128, h_{2}=256$ and $\left.h_{3}=512\right)$ in the case of data with 5 and 15 minutes resolution. The FCNN models were trained using Stochastic Gradient Descending (SGD) [17]. More specifically, the Adaptive Moment Estimation (ADAM) [18] was chosen to optimize the parameters. Kingma et al. [18] showed that due to bias-correction ADAM slightly outperforms RMSprop and Adadelta towards the end of optimization since the gradients become sparser. The momentum was set to 0.9.

Recently, the rectified linear unit (ReLU) [19] became the most common choice of activation function, defined as $f(x)=\max (0, x)$, for deep learning methods [20]. Compared to sigmoid or tangent functions, ReLU and its variations, allow for faster and effective training of neural networks architectures on large datasets [21]. Thus, we have chosen ReLU as an activation function in all the experiments. Finally, as a convergence criterion for FCNN a maximum limit of 200 iterations was set.

For LSTM, the settings described in [22] were used as a starting point for our implementation. If not stated otherwise, default settings in Python/Keras are used. SVM was set with a linear kernel function, $C=1$ and $\gamma=1$.

\section{Prediction results}

In Figure 3 we can see the difference between true values versus the predicted values using the full-connected neural network method. A summary of these results using all methods can be seen in terms of MSE in Table II and in therms of MAE in Table III.

LSTM shows the best performance in 7 cases from 9 in terms of both MSE and MAE. Decision tree was the most accurate method in terms of MSE in the case of predicting the building energy consumption with PV generation using data with 5 and 15 minutes resolution (second column, Table II),

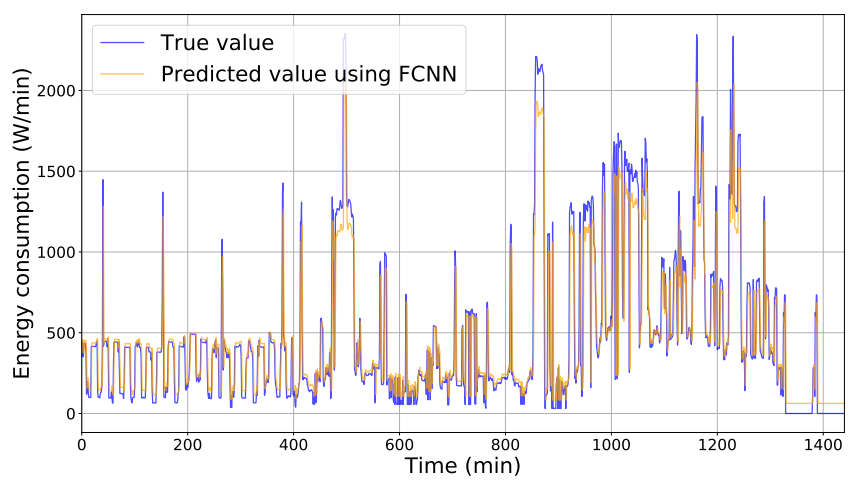

Fig. 3. Building energ prediction results using FCNN method and data with one minte resolution

TABLE II

MEAN SQUARE ERROR (MSE) FOR BUILDING 1 AND 2 ENERGY PREDICTION, AS WELL AS FOR AGGREGATED ELECTRICAL ENERGY CONSUMPTION.

\begin{tabular}{ccccc} 
Resolution & Method & $\begin{array}{c}\text { Building } \\
\text { (without PV) }\end{array}$ & $\begin{array}{c}\text { Building } \\
\text { (with PV) }\end{array}$ & $\begin{array}{c}\text { Aggregated } \\
\text { buildings }\end{array}$ \\
\hline $1 \mathrm{~min}$ & LSTM & 0.0338 & 0.0421 & 0.0473 \\
& FCNN & 0.0339 & 0.0460 & 0.0488 \\
& DT & 0.0358 & 0.0468 & 0.0501 \\
\hline $5 \mathrm{~min}$ & LSTM & 0.0331 & 0.0338 & 0.0425 \\
& FCNN & 0.0337 & 0.0354 & 0.0432 \\
& DT & 0.0359 & 0.0324 & 0.0438 \\
\hline $15 \mathrm{~min}$ & LSTM & 0.0368 & 0.0338 & 0.0385 \\
& FCNN & 0.0395 & 0.0334 & 0.0395 \\
& DT & 0.0399 & 0.0314 & 0.0400
\end{tabular}

TABLE III

MEAN AVREAGE ERROR (MAE) FOR TWO BUILDINGS ENERGY CONSUMPTION WITH AND WITHOUT GENERATION CAPABILITIES, AS WELL AS FOR AGGREGATED ELECTRICAL ENERGY CONSUMPTION.

\begin{tabular}{ccccc} 
Resolution & Method & $\begin{array}{c}\text { Building } \\
{[\%]}\end{array}$ & $\begin{array}{c}\text { Building } \\
\text { (with PV) [\%] }\end{array}$ & $\begin{array}{c}\text { Aggregated } \\
\text { buildings [\%] }\end{array}$ \\
\hline 1 min & LSTM & 12.15 & 11.99 & 15.60 \\
& FCNN & 12.06 & 12.41 & 15.86 \\
& DT & 12.25 & 12.65 & 15.99 \\
\hline 5 min & LSTM & 12.22 & 10.97 & 14.86 \\
& FCNN & 12.41 & 11.55 & 15.25 \\
& DT & 12.71 & 11.33 & 15.34 \\
\hline 15 min & LSTM & 12.67 & 11.20 & 13.70 \\
& FCNN & 13.66 & 11.43 & 14.43 \\
& DT & 13.92 & 10.99 & 14.59
\end{tabular}

while in terms of MAE, FCNN and Decision tree showed the best performance one time (Table III). We may also notice that as the data input to our models are coming with a more fine granularity the error slightly increases for all the methods compared. We will argue that this is a direct effect of the number of time steps predicted in the future, and not necessary a effect of the nonliniarities in the electrical pattern. For example, we predict 1440 time steps with one minute resolution and just 96 time steps in the case of data with 15 minutes resolution. Trying to do a comparison as fair as possible between with the three AI methods we look for a day-ahead prediction.

Thus, as recommended by its performance we have further on kept the LSTM method as the main prediction method 


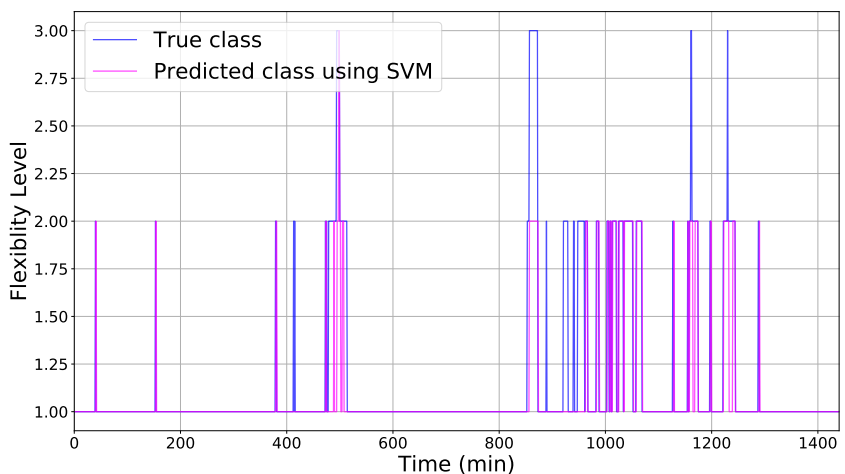

Fig. 4. Estimated flexibility level for aggregated energy using Support Vector Machine (SVM) with one minute resolution.

in the AIdigi platform and we extend it with a classification method for building energy flexibility estimation.

\section{Classification results}

The building energy flexibility is quantified at the aggregated level. A summary of the results are detailed in Table IV and depicted in Figure 4. The overall SVM accuracy is 91,3\%. Even if we have an imbalanced number of points per class the precision and recall assessment metrics showed a good agreement with the accuracy metrics, as it can be observed in Table IV. It is also of major importance that the classification are easily generalized to multi-class contingency tables.

TABLE IV

Classification aCCURACY USING SUPPORT VeCTOR MACHINE (SVM) FOR AGGREGATED BUILDING ENERGY FLEXIBILITY WITH ONE MINUTE RESOLUTION.

\begin{tabular}{lcccc}
$\begin{array}{l}\text { Class } \\
{[\#]}\end{array}$ & $\begin{array}{c}\text { Total number } \\
\text { of instances }[\#]\end{array}$ & Precision & Recall & $F 1$-score \\
\hline$L_{1}$ & 1243 & 0.93 & 0.99 & 0.96 \\
$L_{2}$ & 171 & 0.69 & 0.50 & 0.58 \\
$L_{3}$ & 25 & 1.00 & 0.08 & 0.15 \\
\hline \multicolumn{2}{r}{ Weighted average } & 0.91 & 0.91 & 0.90
\end{tabular}

\section{CONCLUSION}

In this paper, we analyze the opportunity of using deep learning techniques to improve self-regulated capabilities of building energy management systems. We have proposed a digital platform and evaluated its performance using data with fine granularity in near real-time settings. This prof-of-concept setup was designed to perform energy prediction and building flexibility detection. The selected artificial intelligent methods for prediction show at least $85 \%$ accuracy in the case of building energy data with 1,5 and 15 minute resolution, independently of the buildings PV generation capabilities. Further on, we show an overall SVM accuracy of 91,3\% for building flexibility level classification. Most encouraging of all is how easy the AIdigi platform can incorporate future AI models in (near) real-time to explore the benefits at the synergy between built environment and emerging smart grid technologies and applications.

\section{ACKNOWLEDGMENT}

This research has been partly funded by UNICORN project (University campus operating as a self-regulated network) of Dutch Top Sector Energy. The authors would like to thank to our digiEMS partners for providing the building energy dataset. We also thank them for the useful comments received after our solution was integrated in the digiEMS online system.

\section{REFERENCES}

[1] Y. Wang, Q. Chen, T. Hong, and C. Kang, "Review of smart meter data analytics: Applications, methodologies, and challenges," IEEE Transactions on Smart Grid, pp. 1-1, 2018.

[2] B. Yildiz, J. Bilbao, J. Dore, and A. Sproul, "Recent advances in the analysis of residential electricity consumption and applications of smart meter data," Applied Energy, vol. 208, pp. 402 - 427, 2017.

[3] E. Mocanu, P. Nguyen, M. Gibescu, and W. Kling, "Comparison of machine learning methods for estimating energy consumption in buildings," in PMAPS, July 2014, pp. 1-6.

[4] E. Mocanu, P. H. Nguyen, M. Gibescu, and W. L. Kling, "Deep learning for estimating building energy consumption," Sustainable Energy, Grids and Networks, vol. 6, pp. 91-99, 2016.

[5] E. Mocanu, P. H. Nguyen, M. Gibescu, E. M. Larsen, and P. Pinson, "Demand forecasting at low aggregation levels using factored conditional restricted boltzmann machine," in 2016 Power Systems Computation Conference (PSCC), June 2016, pp. 1-7.

[6] W. Kong, Z. Y. Dong, Y. Jia, D. J. Hill, Y. Xu, and Y. Zhang, "Short-term residential load forecasting based on lstm recurrent neural network," IEEE Transactions on Smart Grid, vol. PP, no. 99, pp. 1-1, 2017.

[7] D. L. Marino, K. Amarasinghe, and M. Manic, "Building energy load forecasting using deep neural networks," Proceedings of the 42nd Annual Conference of the IEEE Industrial Electronics Society (IECON), 2016.

[8] P. A. Mynhoff, E. Mocanu, and M. Gibescu, "Statistical learning versus deep learning: performance comparison for building energy prediction methods," in IEEE PES Innovative Smart Grid Technologies Conference Europe (ISGT-Europe), Sept 2018, pp. 1-6.

[9] A. Rahman, V. Srikumar, and A. D. Smith, "Predicting electricity consumption for commercial and residential buildings using deep recurrent neural networks," Applied Energy, vol. 212, pp. 372 - 385, 2018.

[10] N. G. Paterakis, E. Mocanu, M. Gibescu, B. Stappers, and W. van Alst, "Deep learning versus traditional machine learning methods for aggregated energy demand prediction," in IEEE PES Innovative Smart Grid Technologies Conference Europe, Sept 2017, pp. 1-6.

[11] L. Breiman, "Random forests," Machine Learning, vol. 45, no. 1, 2001.

[12] M. Manic, K. Amarasinghe, J. J. Rodriguez-Andina, and C. Rieger, "Intelligent buildings of the future: Cyberaware, deep learning powered, and human interacting," IEEE Industrial Electronics Magazine, vol. 10, no. 4, pp. 32-49, 2016.

[13] C. Cortes and V. Vapnik, "Support-Vector Networks," Mach. Learn., vol. 20, no. 3, pp. 273-297, Sep. 1995.

[14] C. M. Bishop, Pattern Recognition and Machine Learning, 1st ed. Springer, Oct. 2007.

[15] Y. Freund and R. E. Schapire, "A short introduction to boosting," in In Proceedings of the 60th International Joint Conference on Artificial Intelligence. Morgan Kaufmann, 1999, pp. 1401-1406.

[16] F. Chollet, "keras," GitHub repository, 2015. [Online]. Available: https://github.com/fchollet/keras

[17] L. Bottou and O. Bousquet, "The tradeoffs of large scale learning," in Proceedings of the 20th International Conference on Neural Information Processing Systems, ser. NIPS'07, USA, 2007, pp. 161-168.

[18] D. P. Kingma and J. Ba, "Adam: A method for stochastic optimization," in International Conference on Learning Representations (ICLR), 2015.

[19] V. Nair and G. E. Hinton, "Rectified linear units improve restricted boltzmann machines," 2010, pp. 807-814.

[20] Y. LeCun, Y. Bengio, and G. Hinton, "Deep learning," Nature, vol. 521, no. 7553, pp. 436-444, May 2015.

[21] V. Nair and G. E. Hinton, "Rectified linear units improve restricted boltzmann machines," in International Conference on Machine Learning (ICML-10). Omnipress, 2010, pp. 807-814.

[22] T. Ganegedara, "Stock market predictions with lstm in python," 2018. [Online]. Available: https://www.datacamp.com/community/tutorials/ lstm-python-stock-market 